\title{
Front Matter: Volume 10574
}

, "Front Matter: Volume 10574," Proc. SPIE 10574, Medical Imaging 2018: Image Processing, 1057401 (8 May 2018); doi: 10.1117/12.2315755

SPIE. Event: SPIE Medical Imaging, 2018, Houston, Texas, United States 


\title{
PROGRESS IN BIOMEDICAL OPTICS AND IMAGING
}

\section{Medical Imaging 2018}

Image Processing

\author{
Elsa D. Angelini \\ Bennett A. Landman \\ Editors
}

11-13 February 2018
Houston, Texas, United States

Sponsored by

SPIE

Co-sponsored by

DECTRIS Ltd. (Switzerland)

Cooperating Organizations

AAPM-American Association of Physicists in Medicine (United States)

IFCARS - International Foundation for Computer Assisted Radiology and Surgery (Germany)

MIPS-Medical Image Perception Society (United States)

RSNA—Radiological Society of North America (United States)

WMIS-World Molecular Imaging Society

Published by

SPIE

\section{Volume 10574}

Part One of Two Parts 
The papers in this volume were part of the technical conference cited on the cover and title page. Papers were selected and subject to review by the editors and conference program committee. Some conference presentations may not be available for publication. Additional papers and presentation recordings may be available online in the SPIE Digital Library at SPIEDigitalLibrary.org.

The papers reflect the work and thoughts of the authors and are published herein as submitted. The publisher is not responsible for the validity of the information or for any outcomes resulting from reliance thereon.

Please use the following format to cite material from these proceedings:

Author(s), "Title of Paper," in Medical Imaging 2018: Image Processing, edited by Elsa D. Angelini, Bennett A. Landman, Proceedings of SPIE Vol. 10574 (SPIE, Bellingham, WA, 2018) Seven-digit Article CID Number.

ISSN: 1605-7422

ISSN: $2410-9045$ (electronic)

ISBN: 9781510616370

ISBN: 9781510616387 (electronic)

Published by

SPIE

P.O. Box 10, Bellingham, Washington 98227-0010 USA

Telephone +1 3606763290 (Pacific Time) · Fax +1 3606471445

SPIE.org

Copyright @ 2018 , Society of Photo-Optical Instrumentation Engineers.

Copying of material in this book for internal or personal use, or for the internal or personal use of specific clients, beyond the fair use provisions granted by the U.S. Copyright Law is authorized by SPIE subject to payment of copying fees. The Transactional Reporting Service base fee for this volume is $\$ 18.00$ per article (or portion thereof), which should be paid directly to the Copyright Clearance Center (CCC), 222 Rosewood Drive, Danvers, MA 01923. Payment may also be made electronically through CCC Online at copyright.com. Other copying for republication, resale, advertising or promotion, or any form of systematic or multiple reproduction of any material in this book is prohibited except with permission in writing from the publisher. The CCC fee code is $1605-$ $7422 / 18 / \$ 18.00$.

Printed in the United States of America.

Publication of record for individual papers is online in the SPIE Digital Library.

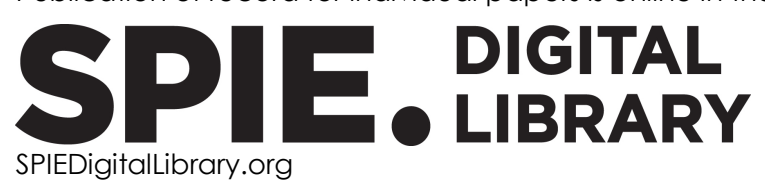

Paper Numbering: Proceedings of SPIE follow an e-First publication model. A unique citation identifier (CID) number is assigned to each article at the time of publication. Utilization of CIDs allows articles to be fully citable as soon as they are published online, and connects the same identifier to all online and print versions of the publication. SPIE uses a seven-digit CID article numbering system structured as follows:

- The first five digits correspond to the SPIE volume number.

- The last two digits indicate publication order within the volume using a Base 36 numbering system employing both numerals and letters. These two-number sets start with 00, 01, 02, 03, $04,05,06,07,08,09,0 A, 0 B \ldots$. OZ, followed by 10-1Z, 20-2Z, etc. The CID Number appears on each page of the manuscript. 


\title{
Contents
}

\author{
xi Authors \\ xvii Conference Committee \\ xxi 2018 Medical Imaging Award Recipients
}

\section{Part One}

1057402 Sulcal depth-based cortical shape analysis in normal healthy control and schizophrenia groups [10574-1]

1057404 Skull segmentation from MR scans using a higher-order shape model based on convolutional restricted Boltzmann machines [10574-3]

1057405 Imaging biomarkers for the diagnosis of Prion disease [10574-4]

1057406 Constructing statistically unbiased cortical surface templates using feature-space covariance [10574-5]

\section{SESSION 2 DEEP LEARNING: SEGMENTATION}

1057407 Segmentation of anatomical structures in cardiac CTA using multi-label V-Net [10574-6]

1057408 Iterative convolutional neural networks for automatic vertebra identification and segmentation in CT images [10574-7]

1057409 Splenomegaly segmentation using global convolutional kernels and conditional generative adversarial networks [10574-8]

10574 OA Segmentation of left ventricle myocardium in porcine cardiac cine MR images using a hybrid of fully convolutional neural networks and convolutional LSTM [10574-9]

10574 OB Towards dense volumetric pancreas segmentation in CT using 3D fully convolutional networks [10574-10]

10574 OC An effective fully deep convolutional neural networks for mitochondria segmentation based on ATUM-SEM [10574-11] 
10574 OD A log-Euclidean and total variation based variational framework for computational sonography [10574-12]

$10574 \mathrm{OE} \quad$ Visualization of coronary artery calcium in dual energy chest radiography using automatic rib suppression [10574-13]

10574 OF Radiation dose reduction in digital breast tomosynthesis (DBT) by means of deep-learningbased supervised image processing [10574-14]

10574 OG Image enhancement method for digital mammography [10574-15]

$10574 \mathrm{OH} \quad$ Image reconstruction using priors from deep learning [10574-16]

10574 Ol Automated abdominal plane and circumference estimation in 3D US for fetal screening [10574-17]

10574 OJ Left ventricle segmentation in 3D ultrasound by combining structured random forests with active shape models [10574-18]

10574 OK Fine segmentation of tiny blood vessel based on fully connected conditional random field [10574-19]

$10574 \mathrm{OL} \quad$ Automatic and fast CT liver segmentation using sparse ensemble with machine learned contexts [10574-20]

10574 OM Nearest neighbor 3D segmentation with context features [10574-21]

10574 ON Detecting multiple myeloma via generalized multiple-instance learning [10574-22]

1057400 A multilevel Markov Chain Monte Carlo approach for uncertainty quantification in deformable registration [10574-23]

10574 OP Quadratic: quality of dice in registration circuits [10574-24]

$10574 \mathrm{OQ}$ Self-reference-based and during-registration detection of motion artifacts in spatiotemporal image data [10574-25]

10574 OR GPU-based stochastic-gradient optimization for non-rigid medical image registration in time-critical applications [10574-26]

10574 OS Deformable image registration using convolutional neural networks [10574-27] 
10574 oU Foveal fully convolutional nets for multi-organ segmentation [10574-29]

10574 OV A novel framework for the local extraction of extra-axial cerebrospinal fluid from MR brain images [10574-30]

10574 OW A statistical model for image registration performance: effect of tissue deformation [10574-31]

\section{SESSION 7 FMRI AND DTI}

10574 OX SHARD: spherical harmonic-based robust outlier detection for HARDI methods [10574-32]

10574 OY Regional autonomy changes in resting-state functional MRI in patients with HIV associated neurocognitive disorder [10574-33]

$10574 \mathrm{OZ}$ Tensor-based vs. matrix-based rank reduction in dynamic brain connectivity [10574-34]

1057410 Extrapolated nonnegative decompositions for the analysis of functional connectivity [10574-35]

1057411 Strain map of the tongue in normal and ALS speech patterns from tagged and diffusion MRI [10574-36]

1057412 TRAFIC: fiber tract classification using deep learning [10574-37]

1057413 Evaluation of inter-site bias and variance in diffusion-weighted MRI [10574-38]

\section{SESSION $8 \quad$ MOTION}

1057414 A novel filtering approach for 3D harmonic phase analysis of tagged MRI [10574-39]

1057415 Feasibility of intra-acquisition motion correction for 4D DSA reconstruction for applications in the thorax and abdomen [10574-40]

1057416 Deep-learning-based CT motion artifact recognition in coronary arteries [10574-41]

1057417 Population-based respiratory 4D motion atlas construction and its application for VR simulations of liver punctures [10574-42]

1057418 Sensitivity analysis of Jacobian determinant used in treatment planning for lung cancer [10574-43]

1057419 HoDOr: histogram of differential orientations for rigid landmark tracking in medical images [10574-44] 
10574 1A Topological leakage detection and freeze-and-grow propagation for improved CT-based airway segmentation [10574-45]

10574 1B Inter-scanner variation independent descriptors for constrained diffeomorphic demons registration of retina OCT [10574-46]

10574 ID Classification of malignant and benign liver fumors using a radiomics approach [10574-48]

10574 IE Quantitative phase and texture angularity analysis of brain white matter lesions in multiple sclerosis [10574-49]

SESSION 10 DEEP LEARNING: LESIONS AND PATHOLOGIES

10574 IF MRI tumor segmentation with densely connected 3D CNN [10574-50]

10574 1G Quantification of lung abnormalities in cystic fibrosis using deep networks [10574-51]

$10574 \mathrm{lH}$ Deep learning for biomarker regression: application to osteoporosis and emphysema on chest CT scans [10574-52]

1057411 Microaneurysm detection using deep learning and interleaved freezing [10574-53]

10574 1J Dataset variability leverages white-matter lesion segmentation performance with convolutional neural network [10574-54]

SESSION 11 DEEP LEARNING: GENERATIVE ADVERSARIAL NETWORKS

10574 1K Modelling the progression of Alzheimer's disease in MRI using generative adversarial networks [10574-55]

10574 IL Learning implicit brain MRI manifolds with deep learning [10574-56]

10574 IM Chest $\mathbf{x}$-ray generation and data augmentation for cardiovascular abnormality classification [10574-57]

$10574 \mathrm{iN}$ Contextual loss functions for optimization of convolutional neural networks generating pseudo CTs from MRI [10574-58]

POSTER SESSION: ENHANCEMENT

1057410 Multi-grid nonlocal techniques for x-ray scatter correction [10574-59]

\section{Part Two}

10574 IP A denoising algorithm for CT image using low-rank sparse coding [10574-60] 
10574 1Q A new method to reduce cone beam artifacts by optimal combination of FDK and TV-IR images [10574-61]

10574 IR CT artifact reduction via U-net CNN [10574-62]

10574 is Automatic segmentation of thoracic aorta segments in low-dose chest CT [10574-63]

10574 IT Fast super-resolution with iterative-guided back projection for 3D MR images [10574-64]

POSTER SESSION: MACHINE LEARNING

$105741 \mathrm{U}$ Automatic localization and segmentation of optical disk based on faster R-CNN and level set in fundus image [10574-65]

10574 IV Circle-like foreign element detection in chest x-rays using normalized cross-correlation and unsupervised clustering [10574-66]

10574 IW Orientation regression in hand radiographs: a transfer learning approach [10574-67]

10574 1X Organ localization and identification in thoracic CT volumes using 3D CNNs leveraging spatial anatomic relations [10574-68]

10574 IY Automatic valve segmentation in cardiac ultrasound time series data [10574-69]

1057412 Transfer learning for diabetic retinopathy [10574-70]

1057420 Extraction of brain tissue from CT head images using fully convolutional neural networks [10574-71]

1057421 Deep learning-based depth estimation from a synthetic endoscopy image training set [10574-72]

1057422 Automatic lung ultrasound B-line recognition in pediatric populations for the detection of pneumonia [10574-73]

1057423 Brain decoding using deep convolutional network and its application in cross-subject analysis [10574-74]

1057424 Neural network fusion: a novel CT-MR aortic aneurysm image segmentation method [10574-75]

1057425 Spine centerline extraction and efficient spine reading of MRI and CT data [10574-76]

POSTER SESSION: QUANTIFICATION AND MODELING

1057426 Segmentation of subcutaneous fat within mouse skin in 3D OCT image data using random forests [10574-77] 
1057427 Automatic detection of the inner ears in head CT images using deep convolutional neural networks [10574-78]

1057428 Multiorgan structures detection using deep convolutional neural networks [10574-79]

1057429 Coupling reconstruction and motion estimation for dynamic MRI through optical flow constraint [10574-80]

10574 2A Sinogram synthesis using convolutional-neural-network for sparsely view-sampled CT [10574-81]

10574 2B High resolution robust and smooth precision matrices to capture functional connectivity [10574-82]

10574 2C Hubs defined with participation coefficient metric altered following acute mTBI [10574-83]

$105742 \mathrm{D}$ Aorta and pulmonary artery segmentation using optimal surface graph cuts in noncontrast CT [10574-84]

10574 2E Model based rib-cage unfolding for trauma CT [10574-85]

$105742 \mathrm{~F}$ Thoracic lymph node station recognition on $\mathrm{CT}$ images based on automatic anatomy recognition with an optimal parent strategy [10574-86]

$105742 \mathrm{G}$ Tapering analysis of airways with bronchiectasis [10574-87]

$105742 \mathrm{H} \quad$ Volumetric versus area-based density assessment: comparisons using automated quantitative measurements from a large screening cohort [10574-88]

105742 Subject-specific brain tumor growth modelling via an efficient Bayesian inference framework [10574-89]

$105742 \mathrm{~J}$ Image-based assessment of uncertainty in quantification of carotid lumen [10574-90]

10574 2K Automated Agatston score computation in non-ECG gated CT scans using deep learning [10574-91]

$105742 \mathrm{~L}$ Generative statistical modeling of left atrial appendage appearance to substantiate clinical paradigms for stroke risk stratification [10574-92]

10574 2M Feature analysis of high SUV regions based on FDG-PET [10574-93]

$105742 \mathrm{~N}$ Relating regional characteristics of left atrial shape to presence of scar in patients with atrial fibrillation [10574-94]

1057420 Automatic anatomy recognition using neural network learning of object relationships via virtual landmarks [10574-117]

10574 2P Training classifiers with limited data using the Radon cumulative distribution transform [10574-118] 
$105742 R \quad$ Enhancement of breast periphery region in digital mammography [10574-96]

1057425 Fast diffeomorphic image registration via GPU-based parallel computing: an investigation of the matching cost function [10574-97]

10574 2T Group-wise shape correspondence of variable and complex objects [10574-98]

POSTER SESSION: SEGMENTATION

$105742 \mathrm{U}$ Student beats the teacher: deep neural networks for lateral ventricles segmentation in brain MR [10574-99]

$105742 \mathrm{~V} \quad$ Fully convolutional neural networks improve abdominal organ segmentation (Cum Laude Poster Award) [10574-100]

10574 2W Multi-class segmentation of neuronal electron microscopy images using deep learning [10574-101]

$105742 X \quad$ Automatic segmentation of fibroglandular tissue in breast MRI using anatomy-driven threedimensional spatial context [10574-102]

$105742 Y \quad$ Extraction of breast lesions from ultrasound imagery: Bhattacharyya gradient flow approach [10574-103]

$105742 Z$ Coupled dictionary learning for joint MR image restoration and segmentation [10574-104]

1057430 Exudate segmentation using fully convolutional neural networks and inception modules [10574-105]

1057431 Deformable model reconstruction of the subarachnoid space [10574-106]

1057432 Convolutional neural network based automatic plaque characterization for intracoronary optical coherence tomography images [10574-107]

1057433 Sequential neural networks for biologically informed glioma segmentation [10574-108]

1057434 A hybrid segmentation method for partitioning the liver based on 4D DCE-MR images [10574-109]

1057435 A new medical image segmentation model based on fractional order differentiation and level set [10574-110]

1057436 Automatic PET cervical tumor segmentation by deep learning with prior information [10574-111]

1057437 Automated segmentation of cellular images using an effective region force [10574-112] 
1057438 Improved stability of whole brain surface parcellation with multi-atlas segmentation [10574-113]

1057439 Feature extraction using convolutional neural networks for multi-atlas based image segmentation [10574-114]

10574 3A Random walk based optic chiasm localization using multi-parametric MRI for patients with pituitary adenoma [10574-115]

10574 3B Advanced two-layer level set with a soft distance constraint for dual surfaces segmentation in medical images [10574-116] 


\section{Authors}

Numbers in the index correspond to the last two digits of the seven-digit citation identifier (CID) article numbering system used in Proceedings of SPIE. The first five digits reflect the volume number. Base 36 numbering is employed for the last two digits and indicates the order of articles within the volume. Numbers start with 00, 01, 02, 03, 04, 05, 06, 07, 08, 09, OA, OB...0Z, followed by 10-12, 20-2Z, etc.

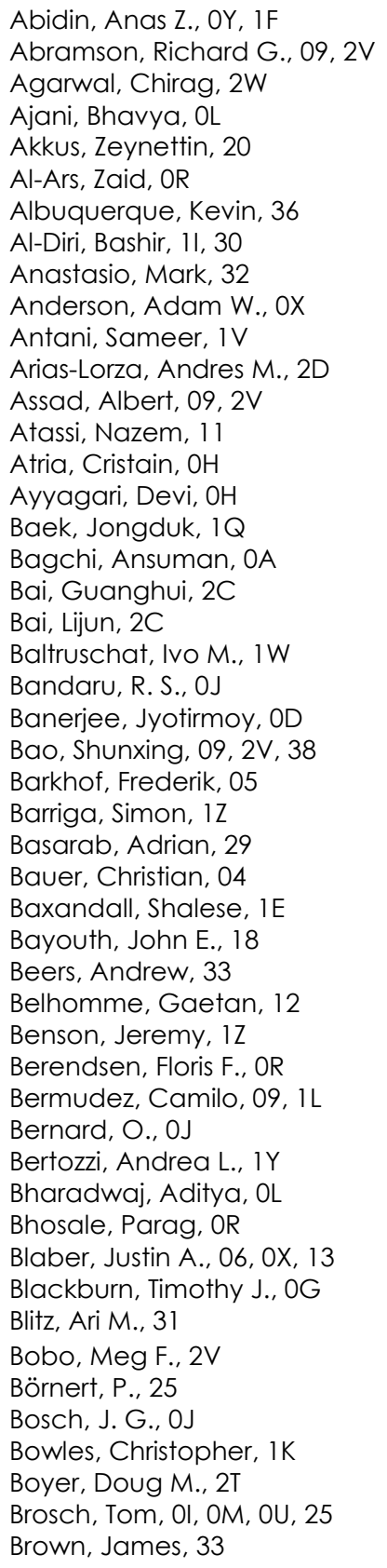

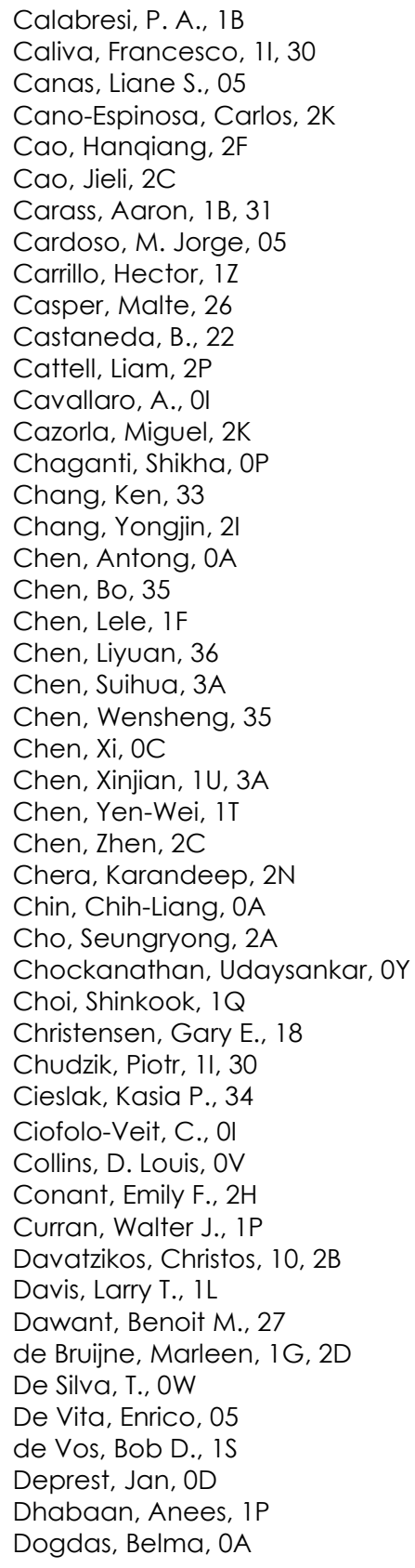


Dong, Xue, IP

Droigk, Christine, 26

DSouza, Adora M., OY, $1 F$

Du, Michael Hong-Fei, 24

Dubost, Florian, $1 \mathrm{G}$

Dukler, Yoni, IY

Durr, Nicholas J., 21

Durumeric, Oguz C., 18

Eche, G., 22

Edmonson, Heidi, 13

Edwards, David, 2G

Ehrhardt, Jan, 00

El Fakhri, Georges, 1 1, 2l

Ellingsen, Lotta M., 31

Elss, T., 16

Eppenhof, Koen A. J., OS

Erickson, Bradley J., 20

Estrada, Trilce, 12

Evers, Michael, 26

Fajardo, Laurie, OF

Fan, Yong, 2Z, 39

Ferrall, Jessica, 12

Fitzgerald, Cailey E., 2P

Folkert, Michael R., 36

Fonov, Vladimir, OV

Forbes, Joseph, OA

Fujiwara, Michitaka, OB

Gan, Shuoqiu, 2C

Gao, Yufei, 23

Gastounioti, Aimilia, 2H

Ge, Yurun, 1Y

Gerard, Sarah E., 18

Gerig, Guido, OV

Gerstner, Elizabeth, 33

Ghafoorian, Mohsen, 2U

Gilkeson, Robert C., OE

Gillard, Jonathan H., 24

Glaister, Jeffrey, 31

Gomez, Arnold D., 14

González Serrano, Germán, 1H, 28, 2K

Grass, M., 16

Gu, Chenghui, 2C

Gu, Wenbo, 29

Gu, Yingying, 10

Gunn, Roger, 1K

Hainline, Allison E., 06, 0X, 13

Hammers, Alexander, $1 \mathrm{~K}$

Han, Hua, OC

Han, R., OW

Han, Xian-Hua, $1 T$

Handels, Heinz, 0O, 0Q, 17, 26

Harari, Colin, 15

Harloff, Andreas, 2J

Harouni, Ahmed El, 07

Hawkes, David J., OD, 2G

Hayashi, Yuichiro, OB

$\mathrm{He}$, Shenghua, 32

$\mathrm{He}, \mathrm{Y}_{\text {., }}$ 1B

Heinrich, Mattias P., OM, IW

Hennemuth, Anja, 2J
Hennig, Juergen, 2J

Hering, Jan, ON

Hilmes, Melissa A., 2V

Hoffman, Eric A., IA

Honnorat, Nicolas, 10, 2B

Hou, Jun, OE

Hristova, Evelin, OM

Hsieh, Meng-Kang, 2H, 2X

$\mathrm{Hu}$, Liuxun, 2C

Huang, Shan, 35

Huang, Yuan, 24

Hugenschmidt, Christina E., $\mathrm{OZ}$

Hunter, Andrew, 11, 30

Hunter, Blake, $1 Y$

Huo, Yuankai, 06, 09, 2V, 38

Hurst, John R., 2G

Icke, Ilknur, OA

Išgum, Ivana, 08, 1S

Iwamoto, Yutaro, $1 \mathrm{~T}$

Jacob, Joseph, 2G

Jacobson, M. W., OW

Jerman, Tim, 1J

Ji, Yuanbo, 3B

Jiang, Yi, OE

Jockel, Sascha, IW

Kalpathy-Cramer, Jayashree, 33

Kang, Hakmook, 02, 06, 0X, 13

Karargyris, Alexandros, $1 \mathrm{M}$

Karssemeijer, Nico, $2 \mathrm{U}$

Kaufhold, Lilli, 2J

Kemner-van de Corput, Mariette, IG

Kepp, Timo, 26

Ketcha, M. D., OW

Khellaf, F., OJ

Khobragade, Nivedita, $2 \mathrm{~W}$

Kim, SunHyung, OV

Kitasaka, Takayuki, OB

Klein, Stefan, 1D

Kleinszig, G., OW

Klinder, Tobias, OI, 2E

Kontos, Despina, $2 \mathrm{H}, 2 \mathrm{X}$

Kostandy, Petro M., 20

Krafft, Axel J., 2J

Krishnan, Karthik, OL

Kybic, Jan, ON

Laeseke, Paul, 15

Lafarge, Maxime W., OS

Lambert, Lukášs, ON

Landman, Bennett A., 02, 06, 09, OP, 0X, 13, 1L, $2 \mathrm{~V}, 38$

Laurienti, Paul J., $0 Z$

Lavini, Cristina, 34

Leclerc, S., OJ

Ledesma Carbayo, María Jesús, 28

Lee, Hoyeon, 2A

Lee, Jongha, 2A

Leeuw, Frank-Erik de, $2 U$

Lefevre, T., 0 ol

Lei, Yang, IP

Lelieveldt, Boudewijn P. F., 3B 
Lenkinski, Robert E., OG

Lessmann, Nikolas, 08

Li, Dandong, 2C

Li, Hua, 32

Li, Lihong, 35

Li, Quanzheng, 21

Li, Shulong, 36

Li, Weifu, OC

Li, Xiang, 31

Liang, Zhengrong, 35

Likar, Boštjan, $1 \mathrm{~J}$

Liò, Pietro, 24

Liu, Jiaqi, 09

Liu, Junchi, OF

Liu, Tian, 1P

Llerena, Rafael, 1 Y

Loog, M., IN

Lorenz, Cristian, Ol, 25, 2E

LU, Qingsheng, 24

Luci, Jeffrey, 13

Lyu, Ilwoo, 02, 06, 0X, 2T, 2V

Ma, Chiyuan, 3A

Madani, Ali, 1M

Madsen, Kristoffer H., 04

Maehara, Akiko, 32

Mahmood, Faisal, 21

Majumdar, Somshubra, 11, 30

Manniesing, Rashindra, $2 \mathrm{U}$

Manstein, Dieter, 26

Maquilan, Genevieve, 36

Marques, Filipe, $1 G$

Mastmeyer, Andre, 17

Mayhugh, Rhiannon E., $\mathrm{OZ}$

Maynard, John, 1 Z

McPhillips, Graeme, $2 \mathrm{G}$

Mead, Simon, 05

Menegatti Pavan, Ana Luiza, 2R

Menon, Prahlad G., 2L, 2N

Miclea, Razvan L., 1D

Mintz, Gary, 32

Misawa, Kazunari, OB

Mistretta, Charles, 15

Modat, Marc, 05

Moeskops, Pim, OS

Mohiuddin, Khadeejah, 37

Mokhtari, Fatemeh, $\mathrm{OZ}$

Moradi, Mehdi, 07, 1M

Mori, Kensaku, OB, OK

Morlock, M., 16

Mostapha, Mahmoud, OV

Mücke, Eike, $0 Q$

Nadeem, Syed Ahmed, IA

Nath, Vishwesh, 0X, 13

Nazarian, Saman, 3B

Nedios, Sotirios, $2 \mathrm{~N}$

Nemeth, Sheila, $1 \mathrm{Z}$

Newton, Allen T., OX, 13, $1 \mathrm{~L}$

Ngattai Lam, Prince D., 12

Nickisch, Hannes, OM, 16, $1 \mathrm{~W}$

Nielsen, Jesper D., 04
Niessen, Wiro J., 1D

Noble, Jack H., 27

Noothout, Julia M. H., 15

O'Connor, Daniel, 29

Oda, Hirohisa, OB

Oda, Masahiro, OB, OK

Odhner, Dewey, 2F, $2 \mathrm{O}$

Oladosu, Michael, $2 \mathrm{~N}$

Onieva Onieva, Jorge, 28

Oshiro, S., 2M

Ourselin, Sébastien, 05, OD

Pan, Yue, 18

Paniagua, Beatriz, 2T

Pantalone, Lauren, $2 \mathrm{H}, 2 \mathrm{X}$

Papageorghiou, A. T., Ol

Parimal, Sarayu, OA

Parvathaneni, Prasanna, 06, 0X, 13, 38

Patel, Premal A., OD

Patterson, Billie, 12

Patton, Taylor J., 18

Patwardhan, Kedar Anil, 19

Pedersen, Jesper Holst, 2D

Peebles, Donald, OD

Perdomo, Jonathan, 2T

Pernuš, Franjo, $1 \mathrm{~J}$

Petean Trindade, Andre, 2R

Philbrick, Kenneth A., 20

Piven, Joseph, OV

Plassard, Andrew J., 09, 1L, 2V

Platel, Bram, $2 \mathrm{U}$

Pluim, Josien P. W., OS

Prasanna, Prasanth, 07

Pridham, Glen, 1E

Prieto, Juan C., 12

Prince, Jerry L., 11 1 14, 1B, 31

Puonti, Oula, 04

Qadir, Ammar, OF

Qian, Yizhou, 1 Y

Quan, Kin, 2G

Quini, Caio Cesar, 2R

Ra, Jong Beom, 2l

Ramesh, Nisha, $\mathrm{OH}$

Ravnik, Domen, $1 \mathrm{~J}$

Raynaud, C., 0 l

Reaungamornrat, S., 1B

Reese, Timothy G., 11

Reinhardt, Joseph M., 18

Rejeski, W. Jack, $0 Z$

Resnick, Susan M., $1 \mathrm{~L}$

Rodrigues de Pina, Diana, $2 R$

Rogers, Baxter P., OX, 13

Rohde, Gustavo K., 2P

Rosen, Bruce, 33

Roth, Holger, OB

Rovet, L., Ol

Roundhill, D., 01

Roy, Snehashis, 31

Ruan, Dan, 29

Rueckert, Daniel, $1 \mathrm{~K}$

Runge, Jurgen H., 34 
Saalbach, Axel, OU, IW

Saha, Punam K., IA

Saidha, S., 1B

Salim, I., Ol

Salma, Nunciada, 26

Sampath, Smita, OA

San José Estépar, Raúl, 1H, 28, 2K

Sanatkhani, Soroosh, 2L, 2N

Sandhu, Romeil, $2 Y$

Santosh, K. C., IV

Schadewaldt, N., 0

Schafer, Sebastian, 15

Schilling, Kurt G., OX

Schmidt-Richberg, A., Ol

Schmitt, H., 16

Schultz, Sandra, 00

Schulz, Heinrich, OM

Schumann, Christian, 2J

Sedghi Gamechi, Zahra, 2D

Seevinck, P. R., IN

Seiler, Stephen, OG

Senda, M., $2 M$

Shackleford, James A., IX

Shao, Meihua, 2C

Shao, Muhan, 31

Shao, Wei, 18

Sharma, Shrushrita, 1E

Sharp, Gregory C., 2

Shen, Chenyang, 36

Shen, Mark D., OV

Sheng, Ke, 29

Shi, Fei, $1 \mathrm{U}$

Shih, Helen A., 2I

Shiino, Akihiko, $1 T$

Shimizu, Natsuki, OB

Shipley, Rebecca J., 2G

Siebner, Hartwig Roman, 04

Sieren, Jered P., IA

Siewerdsen, J. H., OW

Slavine, Nikolai $\vee ., 0 G$

Soans, Rajath Elias, $1 \mathrm{X}$

Soliz, Peter, 1 Z

Speidel, Michael, 15

Špiclin, Žiga, $1 \mathrm{~J}$

Spiga, Filippo, 24

Staring, Marius, OR

Starmans, Martijn P. A., ID

Stoker, Jaap, 34

Stone, Maureen L., 11, 14

Styner, Martin A., OV , 12, 2T

Sudre, Carole, 05

Sun, Chuanzhu, 2C

Sun, Min, 3A

Suzuki, Kenji, OF

Swanson, Meghan, OV

Syeda-Mahmood, Tanveer, 07, IM

Tang, Dalin, 32

Tang, Hui, 07

Tang, Xiaoying, $2 \mathrm{~S}$

Tannenbaum, Allen, $2 Y$
Tanno, Ryutaro, $2 \mathrm{G}$

Tao, Qian, 3B

Tasdizen, Tolga, $\mathrm{OH}$

Teng, Zhongzhao, 24

Tetsuya, T., 2M

Teuwen, Jonas, $2 \mathrm{U}$

Thielscher, Axel, 04

Thomeer, Maarten G., ID

Thornton, John, 05

Tiddens, Harm A. W., IG

Tiwari, Abhishek, 19

Tong, Yubing, 2F, 20

Torigian, Drew A., 2F, $2 \mathrm{O}$

Torigoe, Y., 2M

Torkaman, Mahsa, $2 Y$

Udupa, Jayaram K., 2F, 20

Uneri, A., OW

Ushakov, Fred, OD

Vacavant, Antoine, 2R

van der Geest, Rob J., 3B

van der Voort, Sebastian R., ID

van Ginneken, Bram, 08, 2U

van Gulik, Thomas, 34

Van Leemput, Koen, 04

van Stralen, M., IN

van Vliet, Lucas J., 34

Vavourakis, Vasileios, $2 G$

Vembar, M., 16

Veni, Gopalkrishna, 07

Vercauteren, Tom, OD

Veta, Mitko, OS

Virostko, Jack, 2V

Vogt, N., 25

Vogt, S., OW

von Berg, Jens, 2E

Voorneveld, J. D., OJ

Vos, Frans M., 34

Wagner, Martin, 15

Wan, Justin W. L., 37

Wang, Chenglong, OK

Wang, Duo, 24

Wang, Hongzhi, 07

Wang, Jing, 36

Wang, Shan, 2C

Wang, Tonghe, $1 \mathrm{P}$

Wang, Xiaocui, 2C

Wang, Xiaokai, 14

Washko, George R., 1H, 28, 2K

Wedeen, Van J., 11

Wei, Dong, 2X

Wei, Zheng, OF

Weinstein, Susan, $2 X$

Wen, Di, OE

Werner, René, $0 Q$

Wigdahl, Jeff, $1 \mathrm{Z}$

Wilms, Matthias, 17

Wilson, David L., OE

Wismüller, Axel, OY, IF

Wissel, T., 16

Wolterink, Jelmer M., is 
Woo, Jonghye, 11, 21

Woodward, Neil D., 02, 06

Wozny, P. J., IN

Wu, Jiong, $2 S$

Wu, Xingyu, 2F

Wu, Yue, $1 \mathrm{~F}$

Wu, Zhiyi, 34

Xiao, Chi, OC

Xie, Feifei, 35

Xie, Qiwei, OC

Xing, FangXu, 11

Xing, Y., IR

$X U$, Chenliang, IF

$X U$, Dong, IP

$\mathrm{XU}$, Guoping, 2F, $2 \mathrm{O}$

$X u, H \cup i, 2 C$

$\mathrm{Xu}$, Zhoubing, 09

Xue, Ping, 10

Yamamoto, Shintaro, $1 Y$

Yamamoto, Tokunori, OK

Yan, Fengxia, 20

Yan, Zhihan, 2C

Yang, Limin, OF

Yang, Xiaofeng, 1P

Yang, Xuesong, 2Z, 39

Yao, Li, 23

Yao, Yuang, 09, 2V

Yapuncich, Gabriel S., 2T

Yatsenko, Dimitri, $\mathrm{OH}$

Ye, Limei, 2C

Yen, Jesse T., $1 Y$

Yin, Bo, 2C

Yoshino, Yasushi, OK

Young, Thomas P., 28

Yuan, Baichuan, $1 Y$

Yvernault, Benjamin, 05

Zamora, Gilberto, $1 Z$

Zarshenas, Amin, OF

Zenteno, O., 22

Zhai, Peng, $1 \mathrm{E}$

Zhang, C., IR

Zhang, Defeng, IU

Zhang, Dongqing, 0A, 27

Zhang, Jiacai, 23

Zhang, Jun, 10

Zhang, Rui, 24

Zhang, Tian, 34

Zhang, Yameng, 23

Zhang, Yunyan, $1 \mathrm{E}$

Zhang, Zhiqiang, 3A

Zhao, Heming, $1 U$

Zhao, Long, 1 Y

Zhao, Ningning, 29

Zheng, Jie, 32

Zhou, Bo, OE

Zhou, Wen, 23

Zhou, Y., $1 \mathrm{~N}$

Zhou, Zhengyang, $1 \mathrm{P}$

Zhu, Jin, 24

Zhu, Weifang, $1 \mathrm{U}$ 
Proc. of SPIE Vol. 10574 1057401-16 Downloaded From: https://www.spiedigitallibrary.org/conference-proceedings-of-spie on 26 Apr 2023
Terms of Use: https://www.spiedigitallibrary.org/terms-of-use 


\title{
Conference Committee
}

\author{
Symposium Chairs
}

Leonard Berliner, Weill Cornell Medical College (United States) and New York Presbyterian - Brooklyn Methodist Hospital (United States)

Ronald M. Summers, National Institutes of Health (United States)

Conference Chairs

Elsa D. Angelini, Imperial College London (United Kingdom) and Télécom ParisTech (France)

Bennett A. Landman, Vanderbilt University (United States)

Conference Program Committee

Rafeef Abugharbieh, The University of British Columbia (Canada)

Mostafa Analoui, Livingston Securities LLC (United States)

Brian B. Avants, University of Pennsylvania (United States)

Meritxell Bach-Cuadra, Université de Lausanne (Switzerland)

Kyongtae Ty Bae, University of Pittsburgh Medical Center (United States)

Ulas Bagci, University of Central Florida (United States)

Olivier Colliot, ICM Brain \& Spine Institute (France)

Benoit M. Dawant, Vanderbilt University (United States)

Marleen de Bruijne, Erasmus MC (Netherlands)

Alexandre X. Falcão, Universidade Estadual de Campinas (Brazil)

Aaron Fenster, Robarts Research Institute (Canada)

James Fishbaugh, NYU Tandon School of Engineering (United States)

Alejandro F. Frangi, The University of Sheffield (United Kingdom)

Mona K. Garvin, The University of lowa (United States)

James C. Gee, University of Pennsylvania (United States)

Benjamin Glocker, Imperial College London (United Kingdom)

Miguel Angel González Ballester, Universitat Pompeu Fabra (Spain)

Hayit Greenspan, Tel Aviv University (Israel)

Ghassan Hamarneh, Simon Fraser University (Canada)

David R. Haynor, University of Washington (United States)

Tobias Heimann, Siemens AG (Germany)

Christine P. Hendon, Columbia University (United States)

Ivana Išgum, University Medical Center Utrecht (Netherlands)

Stefan Klein, Erasmus MC (Netherlands)

Ender Konukoglu, ETH Zürich (Switzerland)

Tianhu Lei, MD Imaging Research (United States)

Karim Lekadir, Universitat Pompeu Fabra (Spain) 
Boudewijn P. F. Lelieveldt, Leiden University Medical Center (Netherlands)

Natasha Lepore, The University of Southern California (United States)

Marius George Linguraru, Children's National Medical Center (United States)

Murray H. Loew, The George Washington University (United States)

Cristian Lorenz, Philips Research (Germany)

Frederik Maes, Katholieke Universiteit Leuven (Belgium)

Vincent A. Magnotta, The University of lowa Hospitals and Clinics (United States)

Rashindra Manniesing, Radboud University Medical Center (Netherlands)

Diana Mateus, Technische Universität München (Germany)

Sunanda D. Mitra, Texas Tech University (United States)

Kensaku Mori, Nagoya University (Japan)

Nassir Navab, Technische Universität München (Germany) and Johns Hopkins University (United States)

Mads Nielsen, Niels Bohr Institute (Denmark)

Wiro J. Niessen, Erasmus MC (Netherlands)

Brian Nutter, Texas Tech University (United States)

Sébastien Ourselin, University College London (United Kingdom)

Dzung L. Pham, Henry Jackson Foundation/USU (United States) and National Institutes of Health (United States) and Johns Hopkins University (United States)

Jerry L. Prince, Johns Hopkins University (United States)

Sonia Pujol, Brigham and Women's Hospital (United States)

Xin Qi, Rutgers, The State University of New Jersey (United States)

Punam K. Saha, The University of lowa (United States)

Olivier Salvado, Commonwealth Scientific and Industrial Research Organisation (Australia)

Emine Saritas, Bilkent University (Turkey)

Lin Shi, The Chinese University of Hong Kong (China)

Marius Staring, Leiden University Medical Center (Netherlands)

Martin A. Styner, The University of North Carolina at Chapel Hill (United States)

Raphael Sznitman, Universität Bern (Switzerland)

Philippe Thevenaz, Ecole Polytechnique Fédérale de Lausanne (Switzerland)

Jayaram K. Udupa, University of Pennsylvania (United States)

Koen Van Leemput, Harvard Medical School (United States) and Massachusetts General Hospital (United States)

Tom K. Vercauteren, University College London (United Kingdom)

Tomaž Vrtovec, University of Ljubljana (Slovenia)

Wolfgang Wein, ImFusion GmbH (Germany) 


\section{Session Chairs}

1 Brain: Shapes and Biomarkers

James C. Gee, University of Pennsylvania (United States)

David R. Haynor, University of Washington (United States)

2 Deep Learning: Segmentation

Kensaku Mori, Nagoya University (Japan)

Tom Vercauteren, University College London (United Kingdom)

3 Image Enhancement

Jayaram K. Udupa, University of Pennsylvania (United States)

$4 \quad$ Machine Learning

Punam Kumar Saha, The University of lowa (United States)

5 Registration

Benoit M. Dawant, Vanderbilt University (United States)

Stefan Klein, Erasmus MC (Netherlands)

6 Keynote and Highlights

Murray H. Loew, The George Washington University (United States)

7 fMRI and DTI

Martin A. Styner, The University of North Carolina at Chapel Hill (United States)

8 Motion

Dzung L. Pham, Henry M. Jackson Foundation (United States)

Marc Modat, University College London (United Kingdom)

9 Image Features

Marleen de Bruijne, Erasmus MC (Netherlands)

10 Deep Learning: Lesions and Pathologies

Ivana Išgum, University Medical Center Utrecht (Netherlands)

Cristian Lorenz, Philips Research (Germany)

11 Deep Learning: Generative Adversarial Networks

Mads Nielsen, Niels Bohr Institute (Denmark)

Hayit Greenspan, Tel Aviv University (Israel) 
Proc. of SPIE Vol. 10574 1057401-20 Downloaded From: https://www.spiedigitallibrary.org/conference-proceedings-of-spie on 26 Apr 2023
Terms of Use: https://www.spiedigitallibrary.org/terms-of-use 


\section{Medical Imaging Award Recipients}

\section{Robert F. Wagner Best Student Paper Award}

Robert F. Wagner was an active scientist in the SPIE Medical Imaging meeting, starting with the first meeting in 1972 and continuing throughout his career. He ensured that the BRH, and subsequently the $\mathrm{CDRH}$, was a sponsor for the early and subsequent Medical Imaging meetings, helping to launch and ensure the historical success of the meeting. The Robert F. Wagner All-Conference Best Student Paper Award (established 2014) is acknowledgment of his many important contributions to the Medical Imaging meeting and his many important advances to the field of medical imaging.

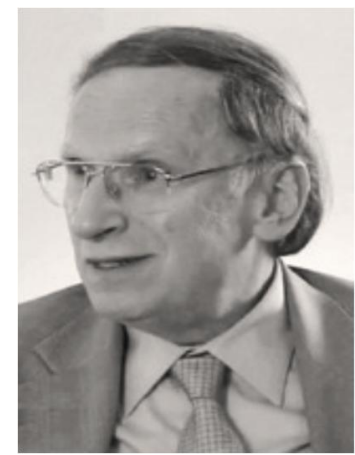

This award is co-sponsored by:

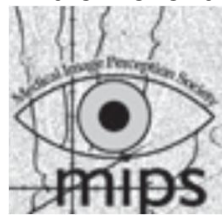

The Medical Image Perception Society

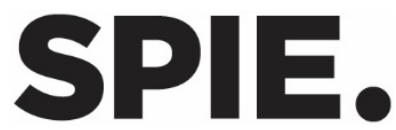

\section{Recipients:}

First Place: Dynamic beam filtering for miscentered patients (10573-29)

Andrew Mao, William Shyr, Grace J. Gang, J. Webster Stayman, Johns Hopkins Univ. (United States)

Second Place: Tumor margin classification of head and neck cancer using hyperspectral imaging and convolutional neural networks (10576-4)

Martin Halicek, Georgia Institute of Technology (United States) and Augusta Univ. (United States); James V. Little, Xu Wang, Emory Univ. School of Medicine (United States); Mihir Patel, Emory Univ. School of Medicine (United States) and The Winship Cancer Institute of Emory Univ. (United States); Christopher C. Griffith, Emory Univ. School of Medicine (United States); Amy Y. Chen, Emory Univ. School of Medicine (United States) and The Winship Cancer Institute of Emory Univ. (United States); Baowei Fei, Georgia Institute of Technology \& Emory Univ. (United States) and The Winship Cancer Institute of Emory Univ. (United States) 
Proc. of SPIE Vol. 10574 1057401-22 Downloaded From: https://www.spiedigitallibrary.org/conference-proceedings-of-spie on 26 Apr 2023
Terms of Use: https://www.spiedigitallibrary.org/terms-of-use 